MIT-CTP-2893

UCLA/99/TEP/27

hep-th/9908160

\title{
Extremal correlators in the AdS/CFT correspondence
}

\author{
Eric D'Hoker ${ }^{a}$, Daniel Z. Freedman ${ }^{b, c}$, Samir D. Mathur ${ }^{b}$ \\ Alec Matusis ${ }^{b}$ and Leonardo Rastelli ${ }^{b}$, \\ a Department of Physics \\ University of California, Los Angeles, CA 90095 \\ and Institute for Theoretical Physics \\ University of California, Santa Barbara, CA 93106 \\ ${ }^{b}$ Center for Theoretical Physics \\ ${ }^{c}$ Department of Mathematics \\ Massachusetts Institute of Technology \\ Cambridge, MA 02139
}

\begin{abstract}
The non-renormalization of the 3-point functions $\left\langle\operatorname{tr} X^{k_{1}} \operatorname{tr} X^{k_{2}} \operatorname{tr} X^{k_{3}}\right\rangle$ of chiral primary operators in $\mathcal{N}=4$ super-Yang-Mills theory is one of the most striking facts to emerge from the AdS/CFT correspondence. A two-fold puzzle appears in the extremal case, e.g. $k_{1}=k_{2}+k_{3}$. First, the supergravity calculation involves analytic continuation in the $k_{i}$ variables to define the product of a vanishing bulk coupling and an infinite integral over AdS. Second, extremal correlators are uniquely sensitive to mixing of the single-trace operators $\operatorname{tr} X^{k}$ with protected multi-trace operators in the same representation of $S U(4)$. We show that the calculation of extremal correlators from supergravity is subject to the same subtley of regularization known for 2-point functions, and we present a careful method which justifies the analytic continuation and shows that supergravity fields couple to single traces without admixture. We
\end{abstract}

\footnotetext{
*e-mails : dhoker@physics.ucla.edu, dzf@math.mit.edu, me@ctpdown.mit.edu, alec.m@ctp.mit.edu, rastelli@ctp.mit.edu
} 
also study extremal $n$-point functions of chiral primary operators, and argue that type IIB supergravity requires that their space-time form is a product of $n-1$ twopoint functions (as in the free-field approximation) multiplied by a non-renormalized coefficient. This non-renormalization property of extremal $n$-point functions is a new prediction of the AdS/CFT correspondence. As a by-product of this work we obtain the cubic couplings $t \phi \phi$ and $s \phi \phi$ of fields in the dilaton and 5 -sphere graviton towers of type IIB supergravity on $A d S_{5} \times S_{5}$. 


\section{Introduction}

The study of correlation functions of operators in the boundary theory is one useful way to explore the AdS/CFT correspondence [1, 2, 3] (see [4] for a comprehensive review). When applied to Type IIB supergravity on $A d S_{5} \times S^{5}$ this study has uncovered several previously unknown properties of the $d=4, \mathcal{N}=4$, super-Yang-Mills (SYM) theory with gauge group $S U(N)$. In the important calculation of [5], 3-point functions of the $\mathcal{N}=4$ chiral primary operators $\operatorname{tr} X^{k} \equiv \operatorname{tr}\left\{X^{i_{1}}(x) X^{i_{2}}(x) \cdots X^{i_{k}}(x)\right\}$ (in the symmetric traceless representation of $S U(4)$ with Dynkin label $(0, k, 0))$ were computed at strong coupling using supergravity, and found to exactly agree at large $N$ with the free field approximation to the SYM theory.

Upon closer examination of the computation of [5], one finds a curious puzzle in the case of 'extremal' 3 -point functions, namely correlators $\left\langle\operatorname{tr} X^{k_{1}} \operatorname{tr} X^{k_{2}} \operatorname{tr} X^{k_{3}}\right\rangle$ in which the conformal dimension of one of the operators is precisely equal to the sum of the other two dimensions, e.g. $k_{1}=k_{2}+k_{3}$. (These values of $k_{i}$ are 'extremal' in the sense that for $k_{1}>k_{2}+k_{3}$ the 3-point function vanishes by $S U(4)$ selection rules.) After dimensional reduction of the field equations of the Type IIB supergravity, scalar fields $s_{k}^{\prime}$ were defined and found to have a simple cubic interaction of the form $\mathcal{G}\left(k_{1}, k_{2}, k_{3}\right) s_{k_{1}}^{\prime} s_{k_{2}}^{\prime} s_{k_{3}}^{\prime}$. In the extremal case $k_{1}=k_{2}+k_{3}$ the coupling $\mathcal{G}\left(k_{2}+k_{3}, k_{2}, k_{3}\right)$ vanishes but the integral over $A d S_{5}$ needed to compute the 3 -point functions diverges [6].

One way around the problem (which was implicitly used in [5] and [7] and that has been recently emphasized in [《]) is to analytically continue in the conformal dimensions $k_{1}, k_{2}$, $k_{3}$. As $k_{1} \rightarrow k_{2}+k_{3}$ the AdS integral computed in [6] diverges as $1 /\left(k_{1}-k_{2}-k_{3}\right)$. In the same limit the supergravity coupling goes to zero as $k_{1}-k_{2}-k_{3}$. Thus by analytic continuation one obtains a finite result for the extremal 3-point functions. This is the answer quoted in [5] which matches the free field result. Although analytic continuation may be regarded as a regularization procedure, it lacks rigourous justification since $S U(4)$ symmetry requires integer dimensions and thus integer $k_{i}$. Additionally, the singularity of the integral over $A d S_{5}$ arises from the region where the bulk interaction point approaches the boundary. In similar situations encountered previously [2, [6] a regularization procedure using a spacetime cutoff inside the boundary was used and found to give satisfactory results. In this case, however, the extremal correlators would vanish for any finite value of the cutoff and also in the limit as the cutoff is removed. Thus the puzzle is to find a method to compute the extremal correlators from first principles, working at exact extremality with definite integer values of $k_{2}, k_{3}, k_{1}=k_{2}+k_{3}$, and we propose such a method in this paper.

A further motivation for considering extremal correlators arises from field theory. It is customarily believed that the supergravity fields $s_{k}^{\prime}$ are dual to the single trace operators $\operatorname{tr} X^{k}$. Indeed both $s_{k}^{\prime}$ and $\operatorname{tr} X^{k}$ belong to the same short representation of the governing 
superalgebra $S U(2,2 \mid 4)$ and have protected scale dimension $\Delta=k$. However, it has been recognized [9, 10, 11], but not widely considered so far, that certain multi-trace operators are also BPS operators with protected dimensions. In addition to the single trace chiral primary $\operatorname{tr}\left\{X^{i_{1}}(x) X^{i_{2}}(x) \cdots X^{i_{k}}(x)\right\}$ one may consider, for example, double trace BPS operators of the form $\operatorname{tr}\left\{X^{i_{1}}(x) X^{i_{2}}(x) \cdots X^{i_{k-l}}(x)\right\} \operatorname{tr}\left\{X^{j_{1}}(x) X^{j_{2}}(x) \cdots X^{j_{l}}(x)\right\}$, in the same $(0, k, 0) S U(4)$ representation. Projection into this representation is obtained by the same process of total symmetrization in the indices $i_{m}$ and removal of traces that is used for $\operatorname{tr} X^{k}$ itself. More generally, one can consider higher order multi-trace operators, schematically denoted by $\operatorname{tr} X^{k_{1}} \operatorname{tr} X^{k_{2}} \cdots \operatorname{tr} X^{k_{m}}$, projected into the representation $(0, k=$ $\left.k_{1}+k_{2}+\cdots k_{m}, 0\right)$. All of these operators transform in the same representation of $S U(2,2 \mid 4)$ as the single trace $\operatorname{tr} X^{k}$, so we would expect these operators to mix, and, indeed, mixing is required by the general structure of operator product expansions in the SYM theory. (The relevance of multi-trace operators for the $A d S_{5} / C F T_{4}$ correspondence on the Coulomb branch has been noted in [12] and 13].)

One is thus naturally led to speculate that supergravity fields couple to linear combinations of single and multiple trace BPS operators. In the free field approximation, one can easily see that operator mixing occurs, although generically suppressed by powers of $1 / N$. Thus one might get the impression that the issue of whether supergravity fields couple to single trace operators or to admixtures with protected multi-traces is a non-leading effect of secondary concern in the AdS/CFT correspondence. However, extremal correlators are exceptional: the contribution of multi-trace operators to extremal 3-point functions is enhanced and of the same order in $N$ as single traces. It is quite curious that multi-trace admixtures are important in exactly the same situation where there is an ambiguity in the calculation of correlation functions. Thus in order to decide whether multi-trace admixtures are present in the AdS/CFT correspondence, it is crucial to have a reliable scheme for computing extremal correlators in supergravity.

In this paper, we will address these issues through a concrete supergravity calculation. We will compute the 3-point functions $\left\langle\mathcal{O}_{t}^{k_{1}} \mathcal{O}_{\phi}^{k_{2}} \mathcal{O}_{\phi}^{k_{3}}\right\rangle$, where $\mathcal{O}_{\phi}^{k}$ and $\mathcal{O}_{t}^{k}$ are the SYM operators that couple respectively to Kaluza-Klein modes of the dilaton $\phi$ and of the supergravity scalar field $t$ (a linear combination of the 4 -form and of the trace of the graviton with indices on the $S^{5}$ ). One motivation for considering this example (which has also been recently studied in [8]) is that the details of the dimensional reduction are somewhat simpler than in the chiral primary computation of [5]. In particular, no subtlety related to the self-duality condition of the 4-form arises, and we will be able to work always at the level of an explicit action. Since the 3-point functions of primary and descendent operators are related by supersymmetry transformations, it is quite clear that our method can be applied to primary correlators also.

It should be noted that two sets of scalar fields were considered in [5]. The dimensional 
reduction procedure initially leads to equations of motion for fields $s_{k}$ with both derivative and non-derivative interactions. A nonlinear transformation to the $s_{k}^{\prime}$ fields then eliminates the derivative couplings. In the descendent sector we work with fields which are analogous to the $s_{k}$ and have both derivative and non-derivative couplings. The interaction Lagrangian is then manipulated by partial integration and use of the linear equations of motion. The value of the on-shell action (as a functional of boundary data) is expressed as the sum of non-derivative cubic bulk coupling plus certain cubic couplings which are total derivatives or boundary interactions. We then compute 3-point correlation functions using the two methods of analytic continuation and space-time cutoff.

We find that in the non-extremal cases $k_{1}<k_{2}+k_{3}$ the contribution of the boundary interactions vanishes as the cutoff is removed, and the net contribution of the bulk vertex is completely unambiguous. At extremality, however, the bulk coupling constants vanish as in the calculation of [5] in the chiral-primary sector. So the cutoff method gives a vanishing bulk contribution, but one of the boundary vertices gives a non-vanishing result. This result is then compared with the limit $k_{1} \rightarrow k_{2}+k_{3}$ of the analytically continued non-extremal correlator, and we find exact agreement.

Our results provide a justification, at least in this example, for the analytic continuation procedure used in [5] and advocated in [8]. We expect a very similar situation to occur in the chiral primary computation of [5]. Namely, the extremal 3-point functions could in principle be obtained from a boundary interaction of the original fields $s_{k}$ that directly arise from the dimensional reduction. In the change of variables to the new fields $s_{k}^{\prime}$ (equ. (3.36) of [5]), these boundary terms are removed and the naive coupling appears to be zero in the extremal case. However, we expect analytic continuation to extremality to give the same answer as a calculation at exact extremality with boundary interactions.

Our analysis thus indicates that the fields $s_{k}$ couple to single trace operators only, although admixtures of multi-trace operators might have been expected. If the new procedure had led to values of extremal correlators different from those obtained from analytic continuation then one would have to add multi-trace admixtures to explain the difference. Since this does not happen, we must conclude that the customary map to single trace operators is correct, or that if present double-traces are suppressed (like $1 / N^{2}$ ).

We also consider extremal $n$-point functions of chiral primary operators. Analysis of the supergravity interactions allows us to prove (under reasonable assumption about the supergravity Lagrangian) that the functional form (that is, the dependence on space-time coordinates) of these correlators in the strong coupling limit is the same as in the free field approximation. Arguments based on the operator product expansion can then be used to show that the coefficient of this functional form satisfies a non-renormalization theorem and is independent of the coupling $\lambda=g^{2} N$.

A curious subtlety emerges from our calculation of extremal 3-point functions. The 
initially derived interaction Lagrangian for the bulk fields is the sum of two bulk vertices, one with two derivatives and one with four derivatives. Each vertex contributes to an extremal 3-point function via a bulk integral which diverges logaritmically as the bulk point $z_{\mu}=\left(z_{0}, z_{i}\right)$ approaces the boundary at $z_{o}=0$. The divergence cancels in the sum of the two integrals, but regularization by a simple cutoff at $w_{0}=\epsilon$ does not give the same result for the correlator as the method described above in which Dirichlet boundary conditions were imposed. Thus extremal 3-point functions are a new example of the subtlety in the regularization of 2-point functions discussed in [3, 6, 13]. Formally 2-point functions, eg $<O_{\phi}^{k_{1}} O_{\phi}^{k_{2}}>$, are also extremal, since $k_{1}=k_{2}$ is required by conformal symmetry.

The paper is organized as follows. In Section 2 we present some results, part new and part review, concerning the mixing of single and multi-trace operators in the SYM field theory. Section 3 contains our treatment of 3 -point correlators in the $t-\phi-\phi$ descendent sector of type $I I B$ supegravity, and Section 4 describes the extension of our ideas to extremal $n$-point functions.

\section{Multi-trace BPS operators and Mixing}

We denote normalized single trace chiral primary operators by

$$
\mathcal{O}_{k}(x)=\frac{1}{N^{k / 2}} \operatorname{tr}\left\{X^{i_{1}}(x) X^{i_{2}}(x) \cdots X^{i_{k}}(x)\right\}
$$

in which it is understood that the tensor is totally symmetric and traceless in the $S U(4)$ indices $i_{\ell}=1, \cdots, 6$. The double-trace product of two such operators $\mathcal{O}_{j}(x) \mathcal{O}_{k-j}(x)$ transforms as the direct sum of the irreducible representations of $S U(4)$ in the tensor product $(0, j, 0) \otimes(0, k-j, 0)$. The tensor product always contains, with unit multiplicity, the representation with Dynkin label $(0, k, 0)$, and the projection into this representation is obtained by symmetrization and removal of traces. Similar remarks apply to the order $m$ multi-trace operator $\mathcal{O}_{k_{1}} \mathcal{O}_{k_{2}} \cdots \mathcal{O}_{k_{m}}$, with $k=k_{1}+k_{2}+\cdots+k_{m}$, which also contains the representation $(0, k, 0)$ with unit multiplicity. We denote these highest weight components of the direct product by

$$
\begin{gathered}
{\left.\left[\mathcal{O}_{k}(x) \mathcal{O}_{\ell}(x)\right]_{\max } \equiv\left[\mathcal{O}_{k}(x) \mathcal{O}_{\ell}(x)\right]\right|_{(0, k+\ell, 0)}} \\
{\left.\left[\mathcal{O}_{k_{1}}(x) \cdots \mathcal{O}_{k_{m}}(x)\right]_{\max } \equiv\left[\mathcal{O}_{k_{1}}(x) \cdots \mathcal{O}_{k_{m}}(x)\right]\right|_{\left(0, k_{1}+\cdots+k_{m}, 0\right)}}
\end{gathered}
$$

There is a simple BPS argument that all of these operators transform in the unique short representation of $S U(2,2 \mid 4)$ whose lowest weight component is a scalar operator in the $(0, k, 0)$ representation of $S U(4)$, of scale dimsnsion $\Delta=k$. Indeed, these operators 
must be lowest weight, since they are totally symmetric in flavor indices, and there can be no appearance of descendents by using the field equations of the interacting SYM theory.

A complete classification of unitary representations of $S U(2,2 \mid 4)$ was given in [14]. For the representations with $S U(4)$ quantum numbers $(0, k, 0)$ of the lowest dimension (scalar) component in the multiplet, two possible types appear : (1) there is a BPS multiplet with dimension $\Delta=k,(2)$ there is a continuous family of representations with $\Delta \geq k+2$. The above operators $\left[\mathcal{O}_{k_{1}} \cdots \mathcal{O}_{k_{m}}\right]$ all have the property that at vanishing $\mathrm{SYM}$ coupling, $\Delta=k$, and thus are in representations of the type (1). Assuming continuity of the dimension as a function of $g$, we see that the operators have to be of type (1) for all values of $g$, and are thus BPS. A somewhat different argument was given in [11.

Next, we show that mixing of single- and double-trace operators is a rigorous consequence of the operator product expansion in conformal field theory. First we observe that the operator product expansion $\mathcal{O}_{k_{2}}(y) \mathcal{O}_{k_{3}}(z)$ contains the (non-leading) term

$$
O_{k_{2}}(y) \mathcal{O}_{k_{3}}(z) \longrightarrow\left[\mathcal{O}_{k_{2}}(y) \mathcal{O}_{k_{3}}(y)\right]_{\max }
$$

with unit coefficient as $z \rightarrow y$, since the operator $\left[\mathcal{O}_{k_{2}}(y) \mathcal{O}_{k_{3}}(y)\right]_{\max }$ is actually defined by the short distance limit. From the $z \rightarrow y$ limit of the extremal 3-point function $\left(k_{1}=k_{2}+k_{3}\right)$

$$
\left\langle\mathcal{O}_{k_{1}}(x) \mathcal{O}_{k_{2}}(y) \mathcal{O}_{k_{3}}(z)\right\rangle=\frac{c}{(x-y)^{2 k_{2}}(x-z)^{2 k_{3}}}
$$

one finds the exact result

$$
\left\langle\mathcal{O}_{k_{1}}(x)\left[\mathcal{O}_{k_{2}}(y) \mathcal{O}_{k_{3}}(y)\right]_{\max }\right\rangle=\frac{c}{(x-y)^{2 k_{1}}}
$$

which shows that the mixed two-point function is non-vanishing and has the same nonrenormalization properties as the extremal 3-point function.

\subsection{Large $N$ counting}

We now apply large $N$ counting arguments to obtain the order in $N$ of the various correlation functions of interest in this paper. To do this one can convert free field Feynman diagrams involving adjoint scalars into fundamental planar 'quark' diagrams in which each closed quark loop gives a factor of $N$. Planar interaction diagrams then have the same net power of $N$ if the 't Hooft coupling $\lambda=g^{2} N$ is fixed. Actually, if the order $\lambda^{2}$ nonrenormalization results of [16] for single-trace correlators and the new results of [11] for correlators of protected multi-trace operators hold in higher order, then free field diagrams tell the whole story. In the formulas below we give the power of $N$ associated with the various correlators of interest in this paper (other factors are usually omitted). 
We normalize free field propagators as

$$
\left\langle X^{a}(x) X^{b}(y)\right\rangle \sim \frac{\delta^{a b}}{(x-y)^{2}}
$$

where $a, b$ are color indices. It then follows that the operators $\mathcal{O}_{k}$ in (2.1) have unit normalization in the large $N$ limit

$$
\left\langle\mathcal{O}_{k}(x) \mathcal{O}_{k}(y)\right\rangle \sim \frac{1}{(x-y)^{2 k}} .
$$

Large $N$ counting then tells us that mixed 2-point functions of single trace operators and protected multi-traces are suppressed by further powers of $N$, specifically (for $k=k_{1}+k_{2}$, etc.)

$$
\begin{array}{r}
\left\langle\mathcal{O}_{k}(x)\left[\mathcal{O}_{k_{1}}(y) \mathcal{O}_{k_{2}}(y)\right]_{\max }\right\rangle \sim \frac{1}{N}, \\
\left\langle\mathcal{O}_{k}(x)\left[\mathcal{O}_{k_{1}}(y) \mathcal{O}_{k_{2}}(y) \ldots \mathcal{O}_{k_{m}}(y)\right]_{\max }\right\rangle \sim \frac{1}{N^{m-1}} .
\end{array}
$$

Thus there is operator mixing even at the free-field level in the SYM theory. The matrix of 2-point functions is diagonalized by eigen-combinations of operators with leading term $\mathcal{O}_{K}$ plus admixtures of $\left[\mathcal{O}_{k_{1}} \mathcal{O}_{k_{2}} \ldots \mathcal{O}_{k_{m}}\right]_{\max }$ with coefficients of order $1 / N^{m-1}$. It is thus suggestive, although logically independent, that supergravity fields $s_{k}$ or $s_{k}^{\prime}$ couple to similar linear combinations in the $A d S_{5} / C F T_{4}$ correspondence, and this hypothesis is explored in the next section.

For 3-point correlators, large $N$ quark loop counting (and $S U$ (4) flavor invariance) give the results

$$
\begin{aligned}
& \left\langle\mathcal{O}_{k_{1}} \mathcal{O}_{k_{2}} \mathcal{O}_{k_{3}}\right\rangle \begin{cases}=0 & \text { if } k_{1}>k_{2}+k_{3} \\
\sim \frac{1}{N} & \text { if } k_{1} \leq k_{2}+k_{3}\end{cases} \\
& \left\langle\left[\mathcal{O}_{l} \mathcal{O}_{k_{1}-l}\right]_{\max } \mathcal{O}_{k_{2}} \mathcal{O}_{k_{3}}\right\rangle \begin{cases}=0 & \text { if } k_{1}>k_{2}+k_{3} \\
\sim 1 & \text { if } k_{1}=k_{2}+k_{3} \text { and } l=k_{2} \text { or } l=k_{3} \\
\sim \frac{1}{N^{2}} & \text { if } k_{1}<k_{2}+k_{3} \text { or } k_{1}=k_{2}+k_{3}, l \neq k_{2}, k_{3} .\end{cases}
\end{aligned}
$$

The last equation shows that the correlators $\left\langle\mathcal{O}_{k_{1}} \mathcal{O}_{k_{2}} \mathcal{O}_{k_{3}}\right\rangle$ and $\left\langle\sum_{l} \frac{1}{N}\left[\mathcal{O}_{l} \mathcal{O}_{k_{1}-l}\right]_{\max } \mathcal{O}_{k_{2}} \mathcal{O}_{k_{3}}\right\rangle$ are comparable in the extremal case $k_{1}=k_{2}+k_{3}$ (and the leading contribution in the second one comes from $l=k_{2}$ or $l=k_{3}$ ), while for $k_{1}<k_{2}+k_{3}$ the second correlator is $O\left(1 / N^{2}\right)$ compared to the first. Thus extremal 3-point functions are special in the sense that double-trace operators give an enhanced contribution. This means that the agreement between supergravity and free-field theory for non-extremal correlators does not test the possible coupling to multi-trace operators, and a reliable method of computation of extremal correlators is required. 
A similar situation holds for (connected) extremal 4-point functions of single and multitrace operators. For example

$$
\left\langle\mathcal{O}_{K} \mathcal{O}_{k_{1}} \mathcal{O}_{k_{2}} \mathcal{O}_{k_{3}}\right\rangle \sim \frac{1}{N^{2}}\left\langle\left[\mathcal{O}_{k_{1}^{\prime}} \mathcal{O}_{k_{2}^{\prime}} \mathcal{O}_{k_{3}^{\prime}}\right]_{\max } \mathcal{O}_{k_{1}} \mathcal{O}_{k_{2}} \mathcal{O}_{k_{3}}\right\rangle \sim \begin{cases}1 & \text { if } k_{i}^{\prime}=k_{i} \\ \frac{1}{N^{3}} & \text { if } k_{i}^{\prime} \neq k_{i}\end{cases}
$$

\section{Supergravity computation of $\left\langle\mathcal{O}_{t, s}^{k_{1}} \mathcal{O}_{\phi}^{k_{2}} \mathcal{O}_{\phi}^{k_{3}}\right\rangle$}

In this Section we describe the computation of the correlation functions $\left\langle\mathcal{O}_{t}^{k_{1}} \mathcal{O}_{\phi}^{k_{2}} \mathcal{O}_{\phi}^{k_{3}}\right\rangle$ and $\left\langle\mathcal{O}_{s}^{k_{1}} \mathcal{O}_{\phi}^{k_{2}} \mathcal{O}_{\phi}^{k_{3}}\right\rangle$, where $\mathcal{O}_{\phi}^{k}, \mathcal{O}_{t}^{k}, \mathcal{O}_{s}^{k}$ are the SYM operators coupling respectively to the supergravity scalar fields $\phi^{k}$ (KK modes of the dilaton), $t^{k}$ and $s^{k}$ (scalar KK modes arising from the 4-form and the graviton with indices on the sphere). In principle these correlators are related by supersymmetry to the 3-point functions of chiral primaries computed in [5], although the explicit relation is cumbersome to obtain.

Besides giving some new potentially useful explicit results for these correlators, the computations presented here will allow us to settle some questions of principle that arise for the 'extremal' cases. We will describe in some detail the calculation of $\left\langle\mathcal{O}_{t}^{k_{1}} \mathcal{O}_{\phi}^{k_{2}} \mathcal{O}_{\phi}^{k_{3}}\right\rangle$, giving particular emphasis to the subtleties that arise for the extremal values $k_{1}=k_{2}+k_{3}$. The computation of $\left\langle\mathcal{O}_{s}^{k_{1}} \mathcal{O}_{\phi}^{k_{2}} \mathcal{O}_{\phi}^{k_{3}}\right\rangle$ is completely analogous and we will just quote the result.

\section{$3.1 \quad$ Set-up}

We closely follow [15]. We adopt almost uniformly the conventions introduced in [5], which we recall here for the reader's convenience. However, unlike [15] and [5] we will work with Euclidean signature.

We use latin indices $i, j, k, \ldots$ for the whole 10-dimensional manifold. Indices $\alpha, \beta, \gamma, \ldots$ are $S^{5}$ indices, while $\mu, \nu, \lambda, \ldots$ are $A d S_{5}$ indices. $G_{m n}$ indicates the metric and $g_{m n}$ its background value. We set the $S^{5}$ and $A d S_{5}$ scales to 1 , i.e. we use units such that the Riemann tensor for the background solution takes the form

$$
R_{\mu \lambda \nu \sigma}=-\left(g_{\mu \nu} g_{\lambda \sigma}-g_{\mu \sigma} g_{\lambda \nu}\right), \quad R_{\alpha \gamma \beta \delta}=\left(g_{\alpha \beta} g_{\gamma \delta}-g_{\alpha \delta} g_{\gamma \beta}\right)
$$

We set

$$
\begin{aligned}
& G_{m n}=g_{m n}+h_{m n}, \\
& h_{\alpha \beta}=h_{(\alpha \beta)}+\frac{h_{2}}{5} ; \quad g^{\alpha \beta} h_{(\alpha \beta)}=0, \\
& h_{\mu \nu}=h_{\mu \nu}^{\prime}-\frac{h_{2}}{3} g_{\mu \nu}, \quad h_{\mu \nu}^{\prime}=h_{(\mu \nu)}^{\prime}+\frac{h^{\prime}}{5} g_{\mu \nu} ; \quad g^{\mu \nu} h_{(\mu \nu)}^{\prime}=0, \\
& F=\bar{F}+\delta F, \quad \delta F_{i j k l m}=5 D_{[i} a_{j k l m]} .
\end{aligned}
$$


Here, $\bar{F}$ is the background value of the $F$-field. Following [15], we choose the gauge conditions $D^{\alpha} h_{\alpha \beta}=D^{\alpha} h_{\mu \alpha}=D^{\alpha} a_{\alpha \mu_{1} m_{2} m_{3} m_{4}}=0$. We refer to [15] for a complete discussion of gauge fixing and for the general expansion of the fluctuation in harmonics of the sphere. We will only need

$$
\begin{aligned}
h_{\mu \nu}^{\prime} & =\sum Y^{k} h_{\mu \nu}^{\prime k}, \\
h_{2} & =\sum Y^{k} h_{2}^{k}, \\
a_{\alpha_{1} \alpha_{2} \alpha_{3} \alpha_{4}} & =\sum D^{\alpha} Y^{k} \epsilon_{\alpha \alpha_{1} \alpha_{2} \alpha_{3} \alpha_{4}} b^{k}, \\
\phi & =\sum Y^{k} \phi^{k}
\end{aligned}
$$

The modes $h_{2}^{k}$ and $b^{k}$ have coupled linear equations of motion. The diagonal combinations are [15] [5]

$$
\begin{aligned}
s^{k} & =\frac{1}{20(k+2)}\left[h_{2}^{k}-10(k+4) b^{k}\right], \\
t^{k} & =\frac{1}{20(k+2)}\left[h_{2}^{k}+10 k b^{k}\right]
\end{aligned}
$$

which satisfy

$$
\begin{aligned}
D_{\mu} D^{\mu} s^{k} & =k(k-4) s^{k}, \\
D_{\mu} D^{\mu} t^{k} & =(k+4)(k+8) t^{k} .
\end{aligned}
$$

\subsection{Constraints}

The equations of motion that follow from supergravity yield some constraints between the modes defined above. It was shown in [15] [5] that if we excite the field $h_{2}$ then the constraints force us to also excite a certain amount of the field $h_{\mu \nu}^{\prime}$. The traceless part and the trace part of this latter field are determined as follows

$$
\begin{aligned}
{h^{\prime}}_{(\mu \nu)}^{k} & =D_{(\mu} D_{\nu)}\left(\frac{2}{5(k+1)(k+3)}\left(h_{2}^{k}-30 b^{k}\right)\right) \\
h^{\prime k} & =\frac{16}{15} h_{2}^{k} .
\end{aligned}
$$

As we will see below, the presence of these constraints will be especially important in the analysis of the extremal 3-point functions.

\subsection{Cubic action}

The kinetic term for the dilaton in the 10-dimensional action is

$$
S=\frac{1}{2 \kappa_{10}^{2}} \int d^{10} x \sqrt{G} \frac{1}{2} G^{m n} \partial_{m} \phi \partial_{n} \phi .
$$


The dilaton also occurs through its coupling to the two-form fields and to the axion, but these latter fields will not be excited in our analysis, and it is consistent to ignore these terms in the action. We can also set

$$
h_{\mu \alpha} \equiv 0 \quad h_{(\alpha \beta)} \equiv 0
$$

Then we can expand

$$
\sqrt{G}=\sqrt{g_{1}} \sqrt{g_{2}}\left(1-\frac{1}{3} h_{2}+\frac{1}{2} h^{\prime}+\ldots\right)
$$

where $g_{1}, g_{2}$ indicate the determinant of the background metric on $A d S_{5}$ and $S^{5}$ respectively. We obtain for the dilaton kinetic term the expansion (to cubic order in the fluctuations)

$$
\begin{aligned}
S & =\frac{1}{2 \kappa_{10}^{2}} \int d^{10} x \sqrt{g}\left[\frac{1}{2} D_{\mu} \phi D^{\mu} \phi+\frac{1}{2} D_{\alpha} \phi D^{\alpha} \phi\right. \\
& \left.+\left(\frac{1}{4} h^{\prime}-\frac{4}{15} h_{2}\right) D_{\alpha} \phi D^{\alpha} \phi+\frac{1}{4} h^{\prime} D_{\mu} \phi D^{\mu} \phi-\frac{1}{2} h_{\mu \nu}^{\prime} D^{\mu} \phi D^{\nu} \phi\right] .
\end{aligned}
$$

\subsection{Dimensional reduction}

We will encounter only scalar fields on the $S^{5}$, and we can expand these fields in scalar spherical harmonics. The spherical harmonics are normalised (following [5], Appendix B) such that

$$
\begin{aligned}
\int Y^{k_{1}} Y^{k_{2}} & =z(k) \delta^{k_{1} k_{2}}, \\
\int Y^{k_{1}} Y^{k_{2}} Y^{k_{3}} & =a\left(k_{1}, k_{2}, k_{3}\right)\left\langle C^{k_{1}} C^{k_{2}} C^{k_{3}}\right\rangle,
\end{aligned}
$$

where

$$
\begin{aligned}
z(k) & =\frac{1}{2^{k-1}(k+1)(k+2)} \\
a\left(k_{1}, k_{2}, k_{3}\right) & =\frac{\omega_{5}}{(\Sigma+2) ! 2^{\Sigma-1}} \frac{k_{1} ! k_{2} ! k_{3} !}{\alpha_{1} ! \alpha_{2} ! \alpha_{3} !} .
\end{aligned}
$$

Here $\alpha_{1}=\frac{1}{2}\left(k_{2}+k_{3}-k_{1}\right), \alpha_{2}=\frac{1}{2}\left(k_{1}+k_{3}-k_{2}\right), \alpha_{3}=\frac{1}{2}\left(k_{1}+k_{2}-k_{3}\right), \Sigma=\frac{1}{2}\left(k_{1}+k_{2}+k_{3}\right)$ and $\omega_{5}=\pi^{3}$ is the area of a unit 5 -sphere. Note that for notational simplicity we omit to indicate explicitly that each field is an element of a vector space of harmonics - we simply indicate the value of $k$. We will also not explicitly write the group-theoretic factors $\left\langle C^{k_{1}} C^{k_{2}} C^{k_{3}}\right\rangle$ in the equations below,

To compute the 3-point functions $\left\langle\mathcal{O}_{t}^{k_{1}} \mathcal{O}_{\phi}^{k_{2}} \mathcal{O}_{\phi}^{k_{3}}\right\rangle$ we need to consider excitations where the fields $s^{k}$ are set to zero. Then from the definition (3.10) of $s^{k}$ and the constraints we 
find

$$
\begin{aligned}
h_{2}^{k} & =10(k+4) b^{k} \\
h_{\mu \nu}^{\prime} & =h_{(\mu \nu)}^{\prime}+\frac{h^{\prime}}{5} g_{\mu \nu}=\frac{2}{5(k+3)(k+4)} D_{\mu} D_{\nu} h_{2}+\frac{2}{15} \frac{k}{k+3} h_{2} g_{\mu \nu} \\
h_{2}^{k} & =10(k+4) t^{k}
\end{aligned}
$$

We then get for the dimensionally reduced form of the action (3.19)

$$
\begin{aligned}
S & =\frac{1}{2 \kappa_{5}^{2}} \int d^{5} x \sqrt{g_{1}}\left[\frac{z(k)}{2}\left(D_{\mu} \phi^{k} D^{\mu} \phi^{k}-k(k+4) \phi^{k} \phi^{k}\right)\right. \\
& \left.+a\left(k_{1}, k_{2}, k_{3}\right)\left(2 \frac{\left(k_{1}+4\right)^{2}}{\left(k_{1}+3\right)} t^{k_{1}} D_{\mu} \phi^{k_{2}} D^{\mu} \phi^{k_{3}}-\frac{2}{k_{1}+3} D_{\mu} D_{\nu} t^{k_{1}} D^{\mu} \phi^{k_{2}} D^{\nu} \phi^{k_{3}}\right)\right]
\end{aligned}
$$

Note that the term $\left(\frac{1}{4} h^{\prime}-\frac{4}{15} h_{2}\right)$ is zero by the constraint equation (3.15), so that we have no 'direct' coupling to the $h_{2}$ field; there is however an 'indirect' coupling given through the excitation of the field $h_{\mu \nu}^{\prime}$. The gravitational coupling constant in (3.27) is related to the SYM parameter $N$ by

$$
2 \kappa_{5}^{2}=\frac{8 \pi^{2}}{N^{2}}
$$

\subsection{Evaluation of the action}

The first cubic term in (3.27) can be manipulated as follows

$$
\begin{aligned}
& \int_{\mathrm{AdS}_{5}} t^{k_{1}} D_{\mu} \phi^{k_{2}} D^{\mu} \phi^{k_{3}}=\int_{\mathrm{AdS}_{5}} t^{k_{1}} \frac{1}{2} D^{\mu} D_{\mu}\left(\phi^{k_{2}} \phi^{k_{3}}\right)-\frac{1}{2}\left(m_{\phi}^{2}\left(k_{2}\right)+m_{\phi}^{2}\left(k_{3}\right)\right) t^{k_{1}} \phi^{k_{2}} \phi^{k_{3}} \\
& =\int_{\mathrm{AdS}_{5}} \frac{1}{2}\left(m_{t}^{2}\left(k_{1}\right)-m_{\phi}^{2}\left(k_{2}\right)-m_{\phi}^{2}\left(k_{3}\right)\right) t^{k_{1}} \phi^{k_{2}} \phi^{k_{3}}+\frac{1}{2} \int_{\partial\left(\mathrm{AdS}_{5}\right)} t^{k_{1}} D_{n}\left(\phi^{k_{2}} \phi^{k_{3}}\right) \\
& -\frac{1}{2} \int_{\partial\left(\mathrm{AdS}_{5}\right)} \phi^{k_{2}} \phi^{k_{3}} D_{n} t^{k_{1}}
\end{aligned}
$$

where we have used the equation of motion for the fields at the linear level. $D_{n}$ indicates the outward normal derivative to the boundary and we have introduced the symbols $m_{\phi}^{2}(k)$ and $m_{t}^{2}(k)$ to denote the masses of the fields $\phi^{k}, t^{k}$

$$
m_{\phi}^{2}(k) \equiv k(k+4) \quad m_{t}^{2}(k) \equiv(k+4)(k+8) .
$$

We now observe that the boundary integrals found in the last step in (3.29) cannot contribute to the 3 -point function if all the three points are disjoint $巴$. This is the case because

$$
\int_{\partial\left(\mathrm{AdS}_{5}\right)} t^{k_{1}} D_{n} \phi^{k_{2}} \phi^{k_{3}}=\iint_{\partial\left(\mathrm{AdS}_{5}\right)} t^{k_{1}}(x) \frac{\partial}{\partial z_{n}} K\left(z, x^{\prime}\right) \phi^{k_{2}}\left(x^{\prime}\right) \phi^{k_{3}}(x) d x d x^{\prime}
$$

\footnotetext{
${ }^{1}$ We thank Oliver DeWolfe for this observation which also invalidates the form of extremal cubic interaction proposed in Appendix A of [24].
} 
where $K$ is the propagator from a boundary point $x^{\prime}$ to a bulk point $z$, and it is assumed in the above equation that we take the limit $z \rightarrow x$. But now note that if we vary such a contribution to the action with respect to the boundary values of the fields, we will obtain a nonzero value only if two of the points where we consider field variations are coincident (namely, $\mathrm{t}$ he points where the fields $t^{k_{1}}$ and $\phi^{k_{3}}$ are varied). We will be interested only in the values of the correlators for separated points. Thus terms of the form of the boundary integrals in (3.29) will always be dropped.

To analyse the last term in (3.27) we carry out the following steps. We first define

$$
P_{\mu \nu}=\frac{1}{2}\left(D_{\mu} \phi^{k_{2}} D_{\nu} \phi^{k_{3}}+D_{\nu} \phi^{k_{2}} D_{\mu} \phi^{k_{3}}\right)-\frac{1}{2} g_{\mu \nu} D^{\lambda} \phi^{k_{2}} D_{\lambda} \phi^{k_{3}}
$$

which satisfies the relation

$$
D^{\mu} P_{\mu \nu}=\frac{1}{2}\left(m_{\phi}^{2}\left(k_{3}\right) \phi^{k_{3}} D_{\nu} \phi^{k_{2}}+m_{\phi}^{2}\left(k_{2}\right) \phi^{k_{2}} D_{\nu} \phi^{k_{3}}\right) .
$$

Then we find

$$
\begin{gathered}
\int_{\mathrm{AdS}_{5}} D_{\mu} D_{\nu} t^{k_{1}} D^{\mu} \phi^{k_{2}} D^{\nu} \phi^{k_{3}} \\
=\frac{1}{2} \int_{\mathrm{AdS}_{5}}\left[m_{t}^{2}\left(k_{1}\right) t^{k_{1}} D^{\lambda} \phi^{k_{2}} D_{\lambda} \phi^{k_{3}}-D_{\nu} t^{k_{1}} t^{k_{1}}\left(P_{\mu \nu}^{2}\left(k_{2}\right) \phi^{k_{2}} D_{\mu} \phi^{k_{3}}+m_{\phi}^{2}\left(k_{3}\right) \phi^{k_{3}} D_{\mu} \phi^{k_{2}}\right)\right] \\
+\int_{\partial\left(\mathrm{AdS}_{5}\right)} D^{\mu} t^{k_{1}} P_{\mu n}
\end{gathered}
$$

The above expression can be further manipulated by steps similar to those in (3.29). Collecting all contributions, we get

$$
\begin{aligned}
& 2 \kappa_{5}^{2} S_{\text {cubic }}=\int_{\mathrm{AdS}_{5}} a\left(k_{1}, k_{2}, k_{3}\right) t^{k_{1}} \phi^{k_{2}} \phi^{k_{3}} \\
& \cdot\left[\frac{\left(k_{1}+4\right)^{2}}{k_{1}+3}\left(m_{t}^{2}\left(k_{1}\right)-m_{\phi}^{2}\left(k_{2}\right)-m_{\phi}^{2}\left(k_{3}\right)\right)+\frac{1}{2\left(k_{1}+3\right)}\left(\left(m_{\phi}^{2}\left(k_{2}\right)-m_{\phi}^{2}\left(k_{3}\right)\right)^{2}-m_{t}^{4}\left(k_{1}\right)\right)\right]+ \\
& \int_{\partial\left(\mathrm{AdS}_{5}\right)} \frac{a\left(k_{1}, k_{2}, k_{3}\right)}{k_{1}+3}\left(-D_{n} \phi^{k_{3}} D_{\mu} t^{k_{1}} D^{\mu} \phi^{k_{2}}-D_{n} \phi^{k_{2}} D_{\mu} t^{k_{1}} D^{\mu} \phi^{k_{3}}+D_{n} t^{k_{1}} D^{\lambda} \phi^{k_{2}} D_{\lambda} \phi^{k_{3}}\right)
\end{aligned}
$$

Using the explicit expressions for the masses of the fields we finally get

$$
\begin{aligned}
& 2 \kappa_{5}^{2} S_{\text {cubic }}=-8 \frac{(\Sigma+4) \alpha_{1}\left(\alpha_{2}+2\right)\left(\alpha_{3}+2\right)}{\left(k_{1}+3\right)} \int_{\mathrm{AdS}_{5}} a\left(k_{1}, k_{2}, k_{3}\right) t^{k_{1}} \phi^{k_{2}} \phi^{k_{3}}+ \\
& \int_{\partial\left(\mathrm{AdS}_{5}\right)} \frac{a\left(k_{1}, k_{2}, k_{3}\right)}{k_{1}+3}\left(-D_{n} \phi^{k_{3}} D_{\mu} t^{k_{1}} D^{\mu} \phi^{k_{2}}-D_{n} \phi^{k_{2}} D_{\mu} t^{k_{1}} D^{\mu} \phi^{k_{3}}+D_{n} t^{k_{1}} D^{\lambda} \phi^{k_{2}} D_{\lambda} \phi^{k_{3}}\right)
\end{aligned}
$$

\subsection{Computing the $3-$ point function}

There are two kinds of terms in (3.36) - a bulk vertex and a boundary term. We imagine carrying out the evaluation of the action in some large but finite region of AdS space, with 
the values of the fields fixed at the boundary of this region. Varying the value of the action with respect to the appropriate fields at chosen points on the boundary we will obtain the 3-point function.

We will find that the case $k_{1}<k_{2}+k_{3}$ and the case $k_{1}=k_{2}+k_{3}$ (the 'extremal' case) need to be analyzed somewhat differently. In the first case, it is easy to see that the boundary integral vanishes as we take the size of the AdS region to infinity, faster than the expected scaling of the three point function. The bulk integral gives a nonvanishing result, which scales exactly as expected for the 3-point function of the boundary CFT.

In the extremal case, on the other hand, we find that the coefficient of the bulk integral is zero. The boundary term in this case gives a nonzero contribution however, since it scales in exactly the manner expected of the 3-point function. Evaluating this boundary integral needs some care, and we will carry out the evaluation below.

\subsubsection{The three-point function for $k_{1}<k_{2}+k_{3}$}

We define $\mathcal{O}_{\phi}^{k}$ and $\mathcal{O}_{t}^{k}$ as the operators that couple to the boundary value of the supergravity fields $\phi^{k}$ and $t^{k}$ with strength 1 , i.e. we let the source terms in the action be

$$
\int d^{4} x \phi^{k}(x) \mathcal{O}_{\phi}^{k}(x), \quad \int d^{4} x t^{k}(x) \mathcal{O}_{t}^{k}(x)
$$

The bulk integral over $A d S_{5}$ in (3.36) was evaluated in [6], equation (25), for fields of generic dimensions $\Delta_{i}$. It suffices to note that the conformal dimensions $\Delta_{i}$ of $t^{k_{1}}, \phi^{k_{2}}$ and $\phi^{k_{3}}$ are related to the KK levels $k_{i}$ through

$$
\Delta_{1}=k_{1}+8 \quad \Delta_{2}=k_{2}+4 \quad \Delta_{3}=k_{3}+4
$$

Then we find that the three-point function is

$$
\begin{aligned}
&\left\langle\mathcal{O}_{t}^{k_{1}}\left(x_{1}\right) \mathcal{O}_{\phi}^{k_{2}}\left(x_{2}\right) \mathcal{O}_{\phi}^{k_{3}}\left(x_{3}\right)\right\rangle=\frac{1}{2 \kappa_{5}^{2}} \frac{4}{\pi^{4}} \frac{a\left(k_{1}, k_{2}, k_{3}\right)}{x_{12}^{8+2 \alpha_{3}} x_{13}^{8+2 \alpha_{2}} x_{23}^{2 \alpha_{1}}} \\
& \times \frac{(\Sigma+4)\left(\alpha_{2}+2\right)\left(\alpha_{3}+2\right)}{\left(k_{1}+3\right)} \frac{\Gamma\left(\alpha_{1}+1\right) \Gamma\left(\alpha_{3}+4\right) \Gamma\left(\alpha_{2}+4\right) \Gamma(\Sigma+6)}{\Gamma\left(k_{1}+6\right) \Gamma\left(k_{2}+2\right) \Gamma\left(k_{3}+2\right)}
\end{aligned}
$$

Although the above calculation is strictly valid only for the range $k_{1}<k_{2}+k_{3}$, we observe that there is a smooth limit as $k_{1} \rightarrow k_{2}+k_{3}$ :

$$
\begin{aligned}
\lim _{k_{1} \rightarrow k_{2}+k_{3}}\left\langle\mathcal{O}_{t}^{k_{1}}\left(x_{1}\right)\right. & \left.\mathcal{O}_{\phi}^{k_{2}}\left(x_{2}\right) \mathcal{O}_{\phi}^{k_{3}}\left(x_{3}\right)\right\rangle=\frac{1}{2 \kappa_{5}^{2}} \frac{4}{\pi^{4}} \frac{a\left(k_{2}+k_{3}, k_{2}, k_{3}\right)}{x_{12}^{8+2 k_{2}} x_{13}^{8+2 k_{3}}} \\
\times & \frac{\left(k_{2}+3\right)\left(k_{2}+2\right)^{2}\left(k_{3}+2\right)\left(k_{3}+3\right)^{2}\left(k_{2}+k_{3}+4\right)}{\left(k_{2}+k_{3}+3\right)} .
\end{aligned}
$$




\subsubsection{The extremal case $k_{1}=k_{2}+k_{3}$}

In this case the coefficient of the bulk integral in (3.36) vanishes. The contribution comes from the boundary term, which must be regulated and calculated carefully. We introduce a cutoff at $z_{0}=\epsilon$, where $z_{0}, z_{i}$ are the coordinates of the usual upper half-plane metric for AdS, and require that bulk fields satisfy a Dirichlet boundary value problem there. This is a geometrically well defined prescription, and leads to the free solution

$$
t^{k}(z)=\int d^{4} x K_{\Delta}^{\epsilon}(z, x) \bar{t}^{k}(x)
$$

where $K_{\Delta}^{\epsilon}(z, x)$ is the Poisson/Dirichlet kernel for the cutoff space-time and $\bar{t}^{k}(x)$ is the boundary source for $t^{k}(z)$. A similar equation holds for $\phi^{k}(z)$. We must insert the free

solution into 3.27 or 3.36 and obtain the contribution to the action functional which is cubic order in the sources.

A certain difficulty now emerges. Both bulk integrals in (3.27) are convergent for regular boundary data, i.e. smeared sources, but the integral from the interaction vertex with four derivatives diverges for point sources, i.e. $\bar{t}^{k}(x) \sim \delta^{4}(x-\hat{x})$. The divergence comes from the region where $z$ is very close to the insertion point $\hat{x}$ where $K_{\Delta}^{\epsilon}(z, x)$ can be well approximated by its flat space form

$$
\text { () } \frac{\left(z_{0}-\epsilon\right)}{\left[\left(z_{0}-\epsilon\right)^{2}+(z-x)^{2}\right]^{5 / 2}} .
$$

This divergence for point sources is an artefact of the cutoff procedure which would occur for all values of the $k_{i}$, not just extremal cases.

In the Appendix the behavior of the singular integral is studied for smeared sources which are peaked about the final boundary insertion points. It is shown that the point source limit is well defined, i.e. finite and independent of details of the smearing. In particular this means that the contribution to the integral of a small strip $\epsilon<z_{0}<\delta$ vanishes as $\delta \rightarrow 0$.

While the Appendix explains that the integral is well defined, a position space approach does not give an easy way to calculate the 3-point function. Instead we used the Fourier transform, since plane waves may be viewed as particular choices of smeared sources for which both the bulk integrals of 3.27 converge. For example the Fourier transform of $K_{\Delta}^{\epsilon}(z, x)$ is well known to be [2, [0]

$$
K_{\Delta}^{\epsilon}(p)=\frac{z_{0}^{\frac{d}{2}} \mathcal{K}_{\Delta-\frac{d}{2}}\left(p z_{0}\right)}{\epsilon^{\frac{d}{2}} \mathcal{K}_{\Delta-\frac{d}{2}}(p \epsilon)}
$$

where $\mathcal{K}_{\nu}$ is the modified Bessel function of index $\nu$, and $d$ is the boundary dimension of $A d S_{d+1}$. (We will specialize to $d=4$ in our final result for the correlator.) We see that 
$K_{\Delta}^{\epsilon}(p)$ admits derivatives of arbitrary order with respect to $z_{0}$ which have smooth limits to the boundary at $z_{0}=\epsilon$.

The partial integrations of Section 3.5 are valid with plane wave sources, so that in the end it is just the Fourier transform of the boundary interaction in (3.36) that must be calculated. It is easy to see that the derivatives in (3.36) which are parallel to the boundary vanish faster in the eventual limit $\epsilon \rightarrow 0$ than those in the normal direction, so we keep only the dominant $z_{0}$ derivatives. The 3 -point correlator in momentum space is just a product of three $K_{\Delta}^{\epsilon}(p)$ with normal derivatives applied.

We use the asymptotic formula

$$
D_{n} K_{\Delta}^{\epsilon}(p)=\left.z_{0} \frac{\partial}{\partial z_{0}} K_{\Delta}^{\epsilon}(p)\right|_{z_{0}=\epsilon}=(d-\Delta)+\ldots a_{\Delta}(p \epsilon)^{2\left(\Delta-\frac{d}{2}\right)} \ln (p \epsilon)+\ldots
$$

where the first ... refer to positive integer powers of $p$ and the second ... to terms containing $\ln (p \epsilon)$ times higher powers of $p$. The coefficient $a_{\Delta}$ is easily obtained from standard treatments of Bessel functions, but for our purposes it suffices to recall that $a_{\Delta} p^{2\left(\Delta-\frac{d}{2}\right)}$ is the Fourier transform of the correctly normalized expression of the 2-point function (Appendix of [6]), which is

$$
\frac{1}{(x-y)^{2 \Delta}} \frac{(2 \Delta-d) \Gamma(\Delta)}{\pi^{\frac{d}{2}} \Gamma\left(\Delta-\frac{d}{2}\right)} .
$$

The relevant term in the product of three propagators is

$$
\begin{gathered}
D_{n} K_{\Delta_{2}+\Delta_{3}}^{\epsilon}\left(p_{1}\right) D_{n} K_{\Delta_{2}}^{\epsilon}\left(p_{2}\right) D_{n} K_{\Delta_{3}}^{\epsilon}\left(p_{3}\right) \\
=\ldots+\epsilon^{2 \Delta_{2}+2 \Delta_{3}-2 d}\left(d-\Delta_{2}-\Delta_{3}\right) a_{\Delta_{2}} a_{\Delta_{3}} p_{2}^{\Delta_{2}-\frac{d}{2}} \ln \left(p_{2}\right) p_{3}^{\Delta_{3}-\frac{d}{2}} \ln \left(p_{3}\right)+\ldots
\end{gathered}
$$

As in previous $p$-space calculations of 2-point functions we have kept the leading term as $\epsilon \rightarrow 0$ which is non-analytic in both $p_{2}$ and $p_{3}$. An interesting feature of this term is that it depends on $p_{2}$ and $p_{3}$ in a factorized way: it is in fact the Fourier transform of a product of two 2-point functions. The factor $\epsilon^{2 \Delta_{2}+2 \Delta_{3}-2 d}$ provides the correct scaling behavior (namely, $\mathcal{O}\left(\epsilon^{\Delta_{1}+\Delta_{2}+\Delta_{3}-2 d}\right)$ ) for a 3-point function of operators of dimension $\Delta_{2}$, $\Delta_{3}, \Delta_{1}=\Delta_{2}+\Delta_{3}$. Observe that in the non-extremal cases $\Delta_{1}<\Delta_{2}+\Delta_{3}$ the boundary term (3.46) scales too fast to give a contribution.

From (3.46) and (3.45), taking into account the overall coefficient in the boundary term of the action (3.36), we finally get for the 3 -point function

$$
\begin{aligned}
\left\langle\mathcal{O}_{t}^{k_{2}+k_{3}}\left(x_{1}\right)\right. & \left.\mathcal{O}_{\phi}^{k_{2}}\left(x_{2}\right) \mathcal{O}_{\phi}^{k_{3}}\left(x_{3}\right)\right\rangle=\frac{1}{2 \kappa_{5}^{2}} \frac{4}{\pi^{4}} \frac{a\left(k_{2}+k_{3}, k_{2}, k_{3}\right)}{x_{12}^{8+2 k_{2}} x_{13}^{8+2 k_{3}}} \\
& \times \frac{\left(k_{2}+3\right)\left(k_{2}+2\right)^{2}\left(k_{3}+3\right)\left(k_{3}+2\right)^{2}\left(k_{2}+k_{3}+4\right)}{\left(k_{2}+k_{3}+3\right)} .
\end{aligned}
$$

Comparison with the expression (3.40) shows exact agreement. 
One might have thought that a simpler way to calculate the extremal 3-point function is to calculate the two bulk integrals in (3.27) using a simple cutoff at $z_{0}=\epsilon$ with standard bulk-to-boundary propagators [3]

$$
K_{\Delta}(z, x) \sim\left(\frac{z_{0}}{z_{0}^{2}+(z-x)^{2}}\right)^{\Delta} .
$$

Each integral is logarithmically divergent, but the divergence cancels in the sum. The final result depends on how the integrals are cut off, and we discuss two methods. First one can continue $k_{1}$ into the convergent region $k_{1}<k_{2}+k_{3}$ so that each integral contains a pole $1 /\left(k_{2}+k_{3}-k_{1}\right)$ which cancels between them. The final result agrees with (3.48) for non-extremal correlators and its continuation to the extremal case agrees with (3.42). This tests the equivalence of the actions (3.27) and (3.36) in the non-extremal region, but it does not give an independent evaluation of the extremal case. The second method is to cut off each divergent integral at $z_{0}=\epsilon$, do the integrals over the 4 coordinates $z_{i}$, and then observe that the divergent part near $z_{0}=0$ cancels in the integrand when both contributions are combined. The final result then does not agree with the method of analytic continuation. However, the situation of two cancelling divergent integrals is very similar to that of 2-point functions [3, 6, 13, and we believe that it is incorrect to use a simple cutoff without imposing Dirichlet boundary conditions. In the case of 2-point functions it was shown [6] that the supergravity calculation of a 3-point function of a current and two scalar operators is unambiguous. The Ward identity then gave a scalar 2point function which agreed with the calculation by the Dirichlet method. In the present case we suggest that the 4-point function of a current and an extremal combination of scalar operators will also be unambiguous, and the Ward identity will lead to an extremal 3 -point correlator which agrees with (3.47).

\subsection{Discussion}

The agreement of extremal 3-point functions calculated by the two methods of analytic continuation in the scale dimensions $k_{i}$ and carefully regulated boundary interaction is the principal result of this section. The result was obtained in a descendent sector of the theory because it was technically easier to find the interaction Lagrangian. However, the 3 -point correlators of chiral primary operators and their descendents are related by $\mathcal{N}=4$ supersymmetry transformations, so our computations also justify the previous calculation of $\left\langle\mathcal{O}_{k_{1}} \mathcal{O}_{k_{2}} \mathcal{O}_{k_{3}}\right\rangle$ obtained in [5] by (implicit) analytic continuation. The agreement with free field theory results then shows that the fields $s^{k}$ used in [5] couple only to the singletrace operators $\mathcal{O}_{k}$ rather than to admixtures with protected multi-trace operators. A 3-point correlator of one double-trace and two single-trace operators can then be obtained 
within the $A d S_{5} / \mathrm{CFT}_{4}$ correspondence by taking a suitable short distance limit of a 4 point correlators of single traces.

Our calculations involved supergravity fields analogous to the $s^{k}$ of [5], and we chose to process the derivative interactions in (3.27) by partial integration rather than eliminate them by field redefinition of the schematic form $s=s^{\prime}+s^{\prime 2}+\left(\partial_{\mu} s^{\prime}\right)^{2}$ as was done in [5]. We have considered the question of a similar transformation of the fields $t^{k}$ and $\phi^{k}$. For example, for the lowest harmonics $k_{1}=k_{2}=k_{3}=0$, which is an extremal configuration, the transformation

$$
\begin{gathered}
t^{\prime 0}=t^{0}+\frac{a(0,0,0)}{3} D_{\mu} \phi^{0} D^{\mu} \phi^{0} \\
\phi^{\prime 0}=\phi^{0}+\frac{2 a(0,0,0)}{3} D_{\mu} t^{0} D^{\mu} \phi^{0} .
\end{gathered}
$$

takes us directly from (3.27) to an action for $t^{\prime 0}$ and $\phi^{\prime 0}$ which is free through cubic couplings. The equations of motion are not used in this transformation and no boundary terms appear. One might be tempted to say that the new fields couple to mixtures of single- and double-trace operators (as proposed in 21] for $s^{\prime k}$ fields), but we could not demonstrate this to our satisfaction. It may also be the case that the required transformation of fields is inadmissable because it is non-invertible or not compatible with the boundary value problem used in the $A d S / C F T$ correspondence. Frankly, we are still confused about the role of the $s^{\prime k}$ fields, and we suggest that it is an interesting issue for future study.

\section{Non-renormalization conjectures for extremal $n-$ point functions}

Although the considerations of this Section apply to all extremal $n$-point functions of chiral primary operators $\mathcal{O}_{k}$, we discuss the simplest case of 4-point functions in detail and then briefly indicate the general line of argument. We consider correlators $\left\langle\mathcal{O}_{k_{1}} \mathcal{O}_{k_{2}} \mathcal{O}_{k_{3}} \mathcal{O}_{k_{4}}\right\rangle$, with $k_{1}=k_{2}+k_{3}+k_{4}$, as they are computed in the large $N$ limit at strong 't Hooft coupling from the $A d S_{5} / C F T_{4}$ correspondence. We shall now argue that:

i) The structure of the Type IIB supergravity action requires that the space-time form of these correlators is a product of $2-$ point functions, specifically

$$
\left\langle\mathcal{O}_{k_{1}}(x) \mathcal{O}_{k_{2}}(y) \mathcal{O}_{k_{3}}(z) \mathcal{O}_{k_{4}}(w)\right\rangle=\frac{A}{(x-y)^{2 k_{2}}(x-z)^{2 k_{3}}(x-w)^{2 k_{4}}}
$$

where the numerical constant A depends on the scale parameters $k_{i}$. This is the same functional form as in the free-field approximation.

ii) Compatibility of this factored form with operator product expansions requires that the constant $A$ is not renormalized. 
Thus for large $N$, the extremal 4-point functions take the same value at large 't Hooft coupling $\lambda=g_{Y M}^{2} N$ as in the free-field approximation. It is natural to conjecture that the extremal 4-point functions of chiral primary operators at large $N$ are independent of $\lambda=g_{Y M}^{2} N$. As for 3-point functions [5, [16] a stronger version of the conjecture is that these 4-point functions are independent of $g_{Y M}$ for any $N$.

We will take the point of view that the result for the extremal correlator is correctly given by the procedure of analytic continuation in the conformal dimensions $k_{i}$. The result of the previous Section makes us confident that this is indeed the case. It should be possible to recover all of the following analysis by working at exact extremality and carefully considering the boundary-like interactions studied in the previous Section, and we will make some remarks on how we expect this to happen.

We need to consider two types of contributions to $\left\langle\mathcal{O}_{k_{1}} \mathcal{O}_{k_{2}} \mathcal{O}_{k_{3}} \mathcal{O}_{k_{4}}\right\rangle$ : exchange diagrams with two 3-point couplings, and a quartic graph with one 4-point coupling.

Let us begin by examining the exchange diagrams. Without loss of generality we can consider the case where $s_{k_{1}}\left(x_{1}\right)$ and $s_{k_{2}}\left(x_{2}\right)$ join by a cubic vertex to some intermediate field $\phi$, which then joins by a cubic vertex to the other two fields at $x_{3}$ and $x_{4}$. Each of the fields $s_{k_{i}}$ is in $S U(4)$ representation with Dynkin label $\left(0, k_{i}, 0\right)$. Multiplying the representations of the fields at $x_{1}$ and $x_{2}$ and also taking the product of representations for the other pair, is easy to see that the only common representation between these two products has Dynkin label $\left(0, k_{3}+k_{4}, 0\right)$. This must be the representation of the intermediate field $\phi$, and there are two possibilities: either $\phi$ is the primary field $s_{k_{3}+k_{4}}$ or it is a $S U(2,2 \mid 4)$ descendent. By detailed consultation of the Tables in [15] which present the spectrum of the dimensionally reduced Type IIB theory and those of [23] on the structure of the relevant representation of the superalgebra $S U(2,2 \mid 4)$, one learns that the possible descendent states in the same $S U(4)$ representation have dimension $\Delta>k_{3}+k_{4}$ and they are superconformal descendents of chiral primaries of dimension $\tilde{k}>k_{3}+k_{4}$. We now proceed to discuss in turn the exchange diagrams for primaries and descendents.

(a) If the exchanged field $\phi$ is the chiral primary $s_{k}$ of dimension $k=k_{3}+k_{4}$, then we must consider the cubic couplings $\mathcal{G}\left(k_{1}, k_{2}, k\right) s_{k_{1}} s_{k_{2}} s_{k}$ and $\mathcal{G}\left(k_{3}, k_{4}, k\right) s_{k_{3}} s_{k_{4}} s_{k}$. However both vertices are extremal and the coupling constants vanish. Stated in terms of a calculation of the exchange diagram by analytic continuation, we find a double zero in the numerator. However, each of the two integrals over $A d S_{5}$ produces a pole so the net amplitude is finite. One way to see this is to generalize the argument used for the contact graph. In the first step one sees that the integral diverges when the adjacent internal vertex approaches the boundary point $\vec{x}_{1}$ of the highest dimension operator. The singular pole factor $1 / \delta$ of (4.2) multiplies the space-time product of $1 /\left(\vec{x}_{1}-\vec{x}_{2}\right)^{2 k_{2}}$ times a further divergent integral which is exactly the extremal 3-point function of primary operators of dimension $k_{3}, k_{4}$ and $k_{3}+k_{4}$. The net result for the amplitude is a finite multiple of the 
factorized form of (4.3). One can also see that the same result is obtained from the scalar exchange integral computed in Sec. 3c of [24] which has a double pole for the relevant values of scale dimensions of external and exchanged fields.

(b) The treatment of descendent exchange graphs is more complicated. First note [15] that the descendents in question are scalars and symmetric tensor fields in the supergravity theory, so higher spin exchange diagrams which have not yet been studied in general form are involved. The coupling constants for the cubic vertices $\phi s_{k_{1}} s_{k_{2}}$ and $\phi s_{k_{3}} s_{k_{4}}$ are related by supersymmetry to the primary vertices in the previous paragraph except that now we have $\tilde{k}>k_{3}+k_{4}$. The coupling $\mathcal{G}\left(k_{3}, k_{4}, \tilde{k}\right)$ vanishes (by $S U(4)$ flavor symmetry), but $\mathcal{G}\left(k_{1}, k_{2}, \tilde{k}\right)$ does not vanish. Thus only one of the two descendent couplings vanishes. Consider the case of an exchanged scalar descendent field. It can be seen from examination of Sec 3c of [24] that the exchange integral has a single pole for relevant values of the dimensions, and that the singular factor multiplies exactly the space-time function we need. The divergence in this case comes when both interaction vertices are within a small region near the boundary point $\vec{x}_{1}$ of the highest dimension operator. The singular contribution does not depend on the spin of the exchanged field, since the short distance behavior of the bulk-to-bulk propagator is universal. Thus we believe that both descendent scalar and tensor exchange graphs contribute finite multiples ofthe desired factored space-time form (4.3).

Next, we analyse the quartic graph, which (after an appropriate field redefinition) is obtained from a 4 -point vertex of the form

$$
\int_{A d S_{5}} \mathcal{G}\left(k_{1}, k_{2}, k_{3}, k_{4}\right) s_{k_{1}} s_{k_{2}} s_{k_{3}} s_{k_{4}}
$$

The coefficient $\mathcal{G}\left(k_{1}, k_{2}, k_{3}, k_{4}\right)$ of this vertex in the supergravity action is not yet known, although it is certainly possible to obtain it by extending the analysis of [5].

We now observe that the integral over the $A d S_{5}$ space involved in the extremal quartic graph diverges. The divergence comes from the region where the integration variable approaches the location of the operator of higher dimension $\mathcal{O}_{k_{1}}$ on the boundary. To see this divergence, note that near this point the propagator $K_{k_{1}}$ behaves as $\left(\frac{z_{0}}{\left(z-\vec{x}_{1}\right)^{2}}\right)^{k_{1}}$, while each of the other propagators behaves as $\left(z_{0}\right)^{k_{i}}, i=2,3,4$. The integration measure is $\frac{d^{5} z}{z_{0}^{5}}$. Thus we see that the integral is logarithmically divergent at $z \rightarrow \vec{x}_{1}$.

Assembling the results for the quartic term with those of the exchange diagrams, for the case of extremal correlators, we conclude the following. Since the exchange diagrams yield finite contributions, and the AdS integral for the quartic diagram diverges, a finite result for the 4 -point function requires that the quartic coupling vanishes at extremality.

\footnotetext{
${ }^{1}$ Recently, all the cubic couplings between two $s$ fields and any other supergravity field have been explicitly determined [21, 22].
} 
All of the following considerations are subject to this plausible (and in principle checkable) assumption.

To regulate the divergent integral, and find the value of the extremal 4-point function, consider the following procedure of analytic continuation. Let the fields $s_{k_{i}}$, for $i=2,3,4$, have dimension $k_{i}+\delta$, while we keep the dimension of $s_{k_{1}}$ to be $k_{1}$. The integral is then finite, though it is still dominated by the region in the infinitesimal vicinity of $z \rightarrow \vec{x}_{1}$. Thus the propagators $K_{k_{i}}$, for $i=2,3,4$, be approximated by the form

$$
\frac{z_{0}^{k_{i}+\delta}}{\left(\vec{x}_{i}-\vec{x}_{1}\right)^{2 k_{i}}}
$$

The integral thus gives

$$
\prod_{i=2,3,4} \frac{1}{\left(\vec{x}_{i}-\vec{x}_{1}\right)^{2 k_{i}}} \int \frac{d^{5} z}{z_{0}^{5}} z_{0}^{k_{1}+3 \delta}\left(\frac{z_{0}}{\left(z-\vec{x}_{1}\right)^{2}}\right)^{k_{1}} \sim \prod_{i=2,3,4} \frac{1}{\left(\vec{x}_{i}-\vec{x}_{1}\right)^{2 k_{i}}} \frac{1}{\delta}
$$

In line with our assumption that the coupling is zero at exact extremalty, and in analogy with the 3-point case, it is plausible that if we analytically continue the dimensions as above the coupling $\mathcal{G}\left(k_{1}, k_{2}+\delta, k_{3}+\delta, k_{4}+\delta\right) \sim \delta$. Then the quartic graph gives

$$
\sim \delta \frac{1}{\delta} \prod_{i=2,3,4} \frac{1}{\left(\vec{x}_{i}-\vec{x}_{1}\right)^{2 k_{i}}} \sim\left\langle\mathcal{O}_{k_{2}}\left(\vec{x}_{2}\right) \mathcal{O}_{k_{2}}\left(\vec{x}_{1}\right)\right\rangle\left\langle\mathcal{O}_{k_{3}}\left(\vec{x}_{3}\right) \mathcal{O}_{k_{3}}\left(\vec{x}_{1}\right)\right\rangle\left\langle\mathcal{O}_{k_{4}}\left(\vec{x}_{4}\right) \mathcal{O}_{k_{4}}\left(\vec{x}_{1}\right)\right\rangle
$$

which is exactly the factorized form of (4.1).

Instead of the analytic continuation procedure just outlined, it should be possible to carry out a careful analysis at exact extremality. The bulk coupling $\mathcal{G}\left(k_{1}, k_{2}, k_{3}, k_{4}\right)$ vanishes at extremality, but we expect that in the dimensional reduction a boundary interaction of the form $\int_{\partial\left(\mathrm{AdS}_{5}\right)} D_{n} s_{k_{1}} D_{n} s_{k_{2}} D_{n} s_{k_{3}} D_{n} s_{k_{4}}$ is induced through the constraints. It is easy to check (for example by the momentum space analysis of the previous Section) that such an interaction reproduces the factorized functional form (4.3). (Note that as in the case of 3-point functions, this boundary term gives a contribution only at exact extremality, $\left.k_{1}=k_{2}+k_{3}+k_{4}\right)$.

We conclude that the large $N$, strong 't Hooft coupling limit of the extremal 4-point functions of chiral primary operators, computed in supergravity, takes the factorized form of a product of three 2-point functions, as in (4.3). This is the same functional form as in the free field theory approximation, and it thus suggests that extremal 4-point functions are not renormalized; that is they are independent of $\lambda=g_{Y M}^{2} N$, at least for large $N$ ! Assuming the factorized form it is quite remarkable that one can prove non-renormalization by an operator product argument similar to the argument at the beginning of Section 2 . The argument applies to the general extremal correlator $\left\langle\mathcal{O}_{k_{1}} \mathcal{O}_{k_{2}} \mathcal{O}_{k_{3}} \mathcal{O}_{k_{4}}\right\rangle$, but to simplify 
the notation we consider the particular case $k_{1}=6, k_{2}=k_{3}=k_{4}=2$ which we assume has the form

$$
\left\langle\mathcal{O}_{6}(x) \mathcal{O}_{2}(y) \mathcal{O}_{2}(z) \mathcal{O}_{2}(w)\right\rangle=\frac{A}{(x-y)^{4}(x-z)^{4}(x-w)^{4}} .
$$

We will show that $A$ is not renormalized, if the 2 - and 3 -point functions that appear in the argument below are not renormalized.

Consider the operator product expansion $\mathcal{O}_{6}(x) \mathcal{O}_{2}(y)$ which contains

$$
\mathcal{O}_{6}(x) \mathcal{O}_{2}(y) \stackrel{y \rightarrow x}{\longrightarrow} c_{624}^{\prime} \frac{\mathcal{O}_{4}(x)}{(x-y)^{4}}+c_{62(22)}^{\prime} \frac{\left[\mathcal{O}_{2}(x) \mathcal{O}_{2}(x)\right]_{\max }}{(x-y)^{4}}+\ldots
$$

where ... indicates the contribution of operators in representations of $S U(4)$ different from the common representation $(0,4,0)$ in the $\mathcal{O}_{6} \mathcal{O}_{2}$ and $\mathcal{O}_{2} \mathcal{O}_{2}$ direct products, and also operators in the $(0,4,0)$ representation belonging to long multiplets.

The assumed factorized form of the 4-point function (4.4) requires that the only common operators in the $\mathcal{O}_{6} \mathcal{O}_{2}$ and $\mathcal{O}_{2} \mathcal{O}_{2} O P E$ 's are of dimension 4 (and their derivative descendents which are omitted for simplicity). This rules out a possible contribution to the double OPE from non-protected long-multiplet operators.

We also need the forms of several 2- and 3-point functions, namely

$$
\begin{aligned}
\left\langle\mathcal{O}_{4}(x) \mathcal{O}_{4}(y)\right\rangle & =\frac{a_{44}}{(x-y)^{8}}, \\
\left\langle\mathcal{O}_{4}(x)\left[\mathcal{O}_{2}(y) \mathcal{O}_{2}(y)\right]_{\max }\right\rangle & =\frac{a_{4(22)}}{(x-y)^{8}}, \\
\left\langle\left[\mathcal{O}_{2}(x) \mathcal{O}_{2}(x)\right]_{\max }\left[\mathcal{O}_{2}(y) \mathcal{O}_{2}(y)\right]_{\max }\right\rangle & =\frac{a_{(22)(22)}}{(x-y)^{8}} \\
\left\langle\mathcal{O}_{4}(x) \mathcal{O}_{2}(y) \mathcal{O}_{2}(z)\right\rangle & =\frac{c_{422}}{(x-y)^{4}(x-z)^{4}}, \\
\left\langle\mathcal{O}_{6}(x) \mathcal{O}_{2}(y) \mathcal{O}_{4}(z)\right\rangle & =\frac{c_{624}}{(x-y)^{4}(x-z)^{8}}, \\
\left\langle\mathcal{O}_{6}(x) \mathcal{O}_{2}(y)\left[\mathcal{O}_{2}(z) \mathcal{O}_{2}(z)\right]_{\max }\right\rangle & =\frac{c_{62(22)}}{(x-y)^{4}(x-z)^{8}} .
\end{aligned}
$$

All coefficients above are assumed to be non-renormalized as a consequence of the arguments of 15, 16, 11, and the argument of Sec 2 shows that $a_{4(22)}=c_{422}$. Using the OPE (4.5) in the 3-point functions (4.10) and (4.11) one finds the relations

$$
\begin{array}{r}
c_{624}^{\prime} a_{44}+c_{62(22)}^{\prime} a_{4(22)}=c_{624}, \\
c_{624}^{\prime} a_{4(22)}+c_{62(22)}^{\prime} a_{(22)(22)}=c_{62(22)} .
\end{array}
$$

which imply that the OPE coefficients $c_{624}^{\prime}$ and $c_{62(22)}^{\prime}$ are not renormalized.

The final step is to take the limit $y \rightarrow x$ of the 4 -point function (4.4) and use (4.9) to obtain

$$
A=c_{624}^{\prime} c_{422}+c_{62(22)}^{\prime} c_{22(22)}
$$


which shows that $A$ is not renormalized.

These considerations naturally extend to higher point functions. For extremal $n-$ point functions of chiral primaries, all disconnected diagrams necessarily vanish, since they factorize into several connected graphs each of which cannot be an $S U(4)$ singlet. Using arguments similar to the 4-point function case, one finds that connected diagrams yield, after the analytic continuation procedure, a factorized product of $(n-1)$ two-point functions. Therefore, we are led to the same non-renormalization conjectures for the extremal $n$-point functions of chiral primaries for any $n$.

\section{Appendix}

In this Appendix we present arguments that the extremal 3-point functions, evaluated directly from the bulk version of the Type IIB supergravity action of (3.27), are uniquely defined and finite. This method of evaluating the extremal 3-point functions is equivalent to that starting from the pure surface action of (3.36), but it circumvents subtle divergences encountered at intermediate stages when dealing directly with the surface action (3.36).

To exhibit these subtle divergences, we start from the surface action (3.36), which is all that remains at extremality. In evaluating the 3-point functions, we encounter contributions of the type

$$
\int_{\partial A d S} \partial_{z_{0}} K\left(z_{0}, x ; x_{1}\right) \partial_{z_{0}} K\left(z_{0}, x ; x_{2}\right) \partial_{z_{0}} K\left(z_{0}, x ; x_{3}\right) d^{4} x
$$

The 4-dimensional $x$ integral above has singularities at $x=x_{i}, i=1,2,3$. It is not immediately clear how these singularities should be regulated. If the total 3-point function is to be finite, we should probably expect to find Dirac $\delta$-function contributions localized at the points $x_{i}$ which can compensate for the divergences at $x=x_{i}$. Clearly, the regularization of both the surface integral and the $\delta$-functions will be subtle and must be handled with great care. In particular, the presence of the $\delta$-functions will require us to work with smeared out sources.

What we show below is that the bulk integral (3.27) that we start with is itself well defined. We do this by showing that there is no divergence in such an integral from the vicinity of the points $x_{i}$, the rest of the integral is easily seen to be convergent. Having proved this convergence we see that we can truncate the domain of integration of the bulk point $z$ slightly above the boundary. The boundary is at $z_{0}=\epsilon$, and so the bulk integral will be restricted to $z_{0}>\epsilon+\delta$, with the limit $\delta \rightarrow 0$ being taken before the limit $\epsilon \rightarrow 0$. Since the bulk integral is convergent, the contribution to the integral from the region $\epsilon<z-0<\epsilon+\delta$ is ignorable, but this regularisation allows a sensible and well defined integration by parts. The integration variable $x$ on the boundary is now at $z_{0}=\epsilon+\delta$, and so does not collide with the source insertions at $x_{i}$ which are at a value $z_{0}=\epsilon$. As 
mentioned in section 3, the result computed for extremal correlators in this manner agrees with the analytic continuation of the 3-point function in dimensions from the case where the correlator is not extremal.

To see the regularity of the bulk integrals, consider the integrals cubic in the fields in (3.27). It is not hard to check that the first of these integrals is convergent, so we will make some remaks about the second integral, which help towards showing that this latter integral is convergent too. The most significant divergence comes from the integral in the vicinity of the point $x_{1}$, so we work in a ball around $x_{1}$, with $x_{2}$ and $x_{3}$ assumed to be far away.

(1) We start with a regularisation where we have taken the boundary of our space to be at $z_{0}=\epsilon$ rather than at $z_{0}=0$. As noted in section 3 , at short distances (less than the AdS curvature length scale) we must use the boundary to bulk propagator appropriate to flat space, as given in (3.42). Now we note that to derive the 3-point function, we must take a functional derivative with respect to the boundary values of the fields. To avoid singularities associated with a delta function source, we start with a source that is smeared in a region around the insertion points; since we are concerned with the vicinity of $x_{1}$ we just smear the source at this point and do not concern ourselves with the other sources. Let the smeared field have a profile $f(x)$, with

$$
\int f(x) d x=1
$$

It is important that as we shrink the size of the smearing region the result of the integral should cease to depend on the size and shape of the smearing region, and should be sensitive only to the total integral of $f$ given in the above equation.

(2) There are four derivatives in the term of interest. The directions of differentiation are summed indices, and so these derivatives can be in the $z_{0}$ direction or in the $z_{i}$ directions. The strongest potential divergences arise from the case where the derivatives are in the $z_{0}$ direction. The reason is that the term $\phi_{2}$ is given by

$$
\phi_{2}\left(z_{0}, x\right)=K\left(z_{0}, x ; x_{2}\right)
$$

and $K$ is constructed to be a Dirichlet boundary to bulk propagator. Thus for $x \neq x_{2}$

$$
\lim _{z_{0} \rightarrow \epsilon} K\left(z_{0}, x, x_{2}\right)=0
$$

and a more careful analysis will give

$$
\phi_{2}\left(z_{0}, x\right) \sim \partial_{x_{i}} \phi_{2}\left(z_{0}, x\right) \sim\left(z_{0}-\epsilon\right)
$$

for $z_{0} \rightarrow \epsilon$. The above fact improves the convergence of the terms that involve derivatives in the directions $z_{i}$, and so we will concentrate below on the case where all derivatives are in the direction $z_{0}$. 
(3) The term $t^{k_{1}}$ in eq. (3.29) is

$$
t^{k_{1}}\left(z_{0}, x\right)=\int d y K\left(z_{0}, x ; y\right) f(y)
$$

where we will assume that since we are looking at short distances $K$ has the form of the flat space kernel given above. $x, y$ are 4 -dimensional variables, and $f$ is the smearing function introduced above. For $z_{0} \rightarrow \epsilon$, we may expand

$$
\begin{gathered}
\int d y K_{f}\left(z_{0}, x ; y\right) f(y) \\
=\int d y K_{f}\left(z_{0}, x ; y\right)\left[f(x)+(y-x)_{i} f_{, i}(x)+(y-x)_{i}(y-x)_{j} f_{, i j}(x)+\ldots\right]
\end{gathered}
$$

This equals

$$
\alpha f(x)+\beta\left(z_{0}-\epsilon\right)^{2} f_{, i}{ }^{i}(x)+\ldots
$$

where $\alpha, \beta$ are of order unity, and the term of order $z_{0}-\epsilon$ vanishes by the rotational symmetry of $K_{f}$. (This vanishing will be important in what follows.)

Thus when we compute $z_{0}$ derivatives of $\int K f$ we get

$$
\partial_{z_{0}} \int K_{f}\left(z_{0}, x ; y\right) f(y) d y=\left(z_{0}-\epsilon\right) f_{, i}{ }^{i}(x) \rightarrow 0 \text { for } z_{0} \rightarrow \epsilon
$$

(4) Let us define

$$
h\left(z_{0}, x\right) \equiv \partial_{z_{0}} \phi^{k_{2}}\left(z_{0}, x\right) \partial_{z_{0}} \phi^{k_{3}}\left(z_{0}, x\right)
$$

as mentioned above, $h$ is a smooth bounded function in the region near $x_{1}$ that we are studying. Now the integral we are studying has the form

$$
\int h\left(z_{0}, x\right) \partial_{z_{0}}^{2} K\left(z_{0}, x ; y\right) f(y) d z_{0} d x d y
$$

and we are considering the integral in a box in 5-d space, enclosing the point $x_{1}$ of the boundary but not enclosing $x_{2}, x_{3}$. Let us integrate once by parts. Then we get

$$
\int_{\partial} h(\epsilon, x) \lim _{z_{0} \rightarrow \epsilon} \partial_{z_{0}} K_{f}\left(z_{0}, x ; y\right) f(y) d x d y-\int \partial_{z_{0}} h\left(z_{0}, x\right) \partial_{z_{0}} K_{f}\left(z_{0}, x ; y\right) f(y) d z_{0} d x d y
$$

The first term vanishes by what we found above. The second term we integrate by parts again to get

$$
-\int_{\partial} \partial_{z_{0}} h\left(z_{0}, x\right) \lim _{z_{0} \rightarrow \epsilon} K_{f}\left(z_{0}, x ; y\right) f(y) d x d y+\int \partial_{z_{0}}^{2} h\left(z_{0}, x\right) K_{f}\left(z_{0}, x ; y\right) f(y) d z_{0} d x d y
$$

This is

$$
-\int_{\partial} \partial_{z_{0}} h\left(z_{0}, x\right) f(x) d x+\int \partial_{z_{0}}^{2} h\left(z_{0}, x\right) K_{f}\left(z_{0}, x ; y\right) f(y) d z_{0} d x d y
$$


Note that the smeared function must satisfy $\int f(x) d x=1$. Since $h$ is smooth, we may approximate the first term in the above equation to get

$$
-\partial_{z_{0}} h\left(z_{0}, x_{1}\right)+\int \partial_{z_{0}}^{2} h\left(z_{0}, x\right) K_{f}\left(z_{0}, x ; y\right) f(y) d z_{0} d x d y
$$

The bulk integral in the above is now no more singular than the two-derivative term in the initial action(3.29).

Note that we have not used any moments of the smearing function apart from its zeroth moment; this is important because as mentioned above if there was any additional dependence on $f$ then the functional derivative was ill defined, and the result would have to be called regulation dependent.

(5) By what was said at the start of this Appendix, we thus understand how to regulate the boundary terms that arise in any integrations by parts in the calculation of the extremal correlator. Instead of explicitly smearing the source function, we can imagine using a momentum basis to expand functions in a region around $x_{1}$ : This region would have to be much larger than the AdS length scale $R$ for the Fourier transform to make sense, but can be taken to be much smaller than the distance to the other insertions at $x_{2}, x_{3}$. This enables a simple calculation, while avoiding any contact terms that can arise when $x_{1}$ approaches $x_{2}, x_{3}$. The result of this calculation was presented in section 3 .

\section{Acknowledgments}

It is a pleasure to aknowledge useful conversations and correspondence with Massimo Bianchi, Elena Caceres, Marc Grisaru, Juan Maldacena, Robert McNees, Oliver DeWolfe, Mukund Rangamani, Witold Skiba, and Leonard Susskind. E.D'H gratefully acknowledges the hospitality of the Institute for Theoretical Physics in Santa Barbara and of the Aspen Center for Physics, where part of this work was carried out.

The research of E.D'H is supported in part by NSF Grants No. PHY-95-31023 and PHY-98-19686, D.Z.F. by NSF Grant No. PHY-97-22072, S.D.M., A.M. and L.R. by D.O.E. cooperative agreement DE-FC02-94ER40818. L.R. is supported in part by INFN 'Bruno Rossi' Fellowship.

\section{References}

[1] J. Maldacena, 'The Large $N$ Limit of Superconformal Theories and Supergravity', Adv.Theor.Math.Phys. 2 (1998) 231-252, hep-th/9711200.

[2] S.S. Gubser, I.R. Klebanov and A.M. Polyakov, 'Gauge Theory Correlators from Noncritical String Theory', Phys.Lett. B428 (1998) 105-114, hep-th/9802109. 
[3] E. Witten, 'Anti-de Sitter Space and Holography', Adv.Theor.Math.Phys. 2 (1998) 253-291, hep-th/9802150.

[4] O. Aharony, S.S. Gubser, J. Maldacena, H. Ooguri and Y. Oz, "Large N field theories, string theory and gravity," hep-th/9905111.

[5] S. Lee, S. Minwalla, M. Rangamani and N. Seiberg, "Three point functions of chiral operators in D =4, N=4 SYM at large N," Adv. Theor. Math. Phys. 2, 697 (1998) hep-th/9806074.

[6] D.Z. Freedman, S.D. Mathur, A. Matusis and L. Rastelli, "Correlation functions in the CFT(d) / AdS(d+1) correspondence," Nucl. Phys. B546, 96 (1999) hep-th/9804058.

[7] R. Corrado, B. Florea and R. McNees, "Correlation functions of operators and Wilson surfaces in the $\mathrm{d}=6,(0,2)$ theory in the large N limit," hep-th/9902153.

[8] H. Liu and A.A. Tseytlin, "Dilaton - fixed scalar correlators and AdS(5) x S**5 - SYM correspondence," hep-th/9906151.

[9] L. Andrianopoli and S. Ferrara, "On short and long SU(2,2/4) multiplets in the AdS / CFT correspondence," hep-th/9812067.

[10] M. Bianchi, S. Kovacs, G. Rossi and Y.S. Stanev, "On the logarithmic behavior in $\mathrm{N}=4$ SYM theory," hep-th/9906188.

[11] W. Skiba, "Correlators of short multitrace operators in $\mathrm{N}=4$ supersymmetric YangMills," hep-th/9907088.

[12] P. Kraus, F. Larsen and S.P. Trivedi, "The Coulomb branch of gauge theory from rotating branes," JHEP 03, 003 (1999) hep-th/9811120.

[13] I.R. Klebanov and E. Witten, "AdS / CFT correspondence and symmetry breaking," hep-th/9905104.

[14] V.K. Dobrev and V.B. Petkova, "All Positive Energy Unitary Irreducible Representations Of Extended Conformal Supersymmetry," Phys. Lett. 162B, 127 (1985).

[15] H. J. Kim, L. J. Romans, and P. van Nieuwenhuizen, 'The Mass Spectrum Of Chiral $N=2 D=10$ Supergravity on $S^{5}$, Phys. Rev. D32 (1985) 389 .

[16] E. D'Hoker, D.Z. Freedman and W. Skiba, "Field theory tests for correlators in the AdS / CFT correspondence," Phys. Rev. D59, 045008 (1999) hep-th/9807098. 
[17] K. Intriligator, "Bonus symmetries of $\mathrm{N}=4$ superYang-Mills correlation functions via AdS duality," Nucl. Phys. B551, 575 (1999) hep-th/9811047.

[18] K. Intriligator and W. Skiba, "Bonus symmetry and the operator product expansion of N=4 SuperYang-Mills," hep-th/9905020.

[19] B. Eden, P.S. Howe and P.C. West, "Nilpotent invariants in N=4 SYM," hepth/9905085.

[20] A. Petkou and K. Skenderis, "A Nonrenormalization theorem for conformal anomalies," hep-th/9906030.

[21] G. Arutyunov and S. Frolov, "Some cubic couplings in type IIB supergravity on $\operatorname{AdS}(5) \times \mathrm{S}^{* *} 5$ and three point functions in SYM(4) at large N," hep-th/9907085.

[22] S. Lee, "AdS(5) / CFT(4) four point functions of chiral primary operators: Cubic vertices," hep-th/9907108.

[23] M. Gunaydin and N. Marcus, "The Spectrum Of The $S^{5}$ Compactification Of The Chiral N=2, D = 10 Supergravity And The Unitary Supermultiplets Of U(2, 2/4)," Class. Quant. Grav. 2, L11 (1985).

[24] E. D'Hoker and D.Z. Freedman, "General scalar exchange in AdS(d+1)," Nucl. Phys. B550, 261 (1999) hep-th/9811257. 